\title{
Conocimientos y comportamientos sobre factores de riesgo de cáncer de mama en un grupo de mujeres
}

\section{Knowledge and behaviors on breast cancer risk factors among a group of women}

Mtra. Sofía Elena Pérez Zumano•

Ana Laura López Romero.• Luis Ángel Benítez Chavira.•

Mtra. Leticia Sandoval Alonso...

\section{Resumen}

IIntroducción: En México cada 2 horas muere una mujer por cáncer de mama (CaMa), enfermería debe asumir un rol protagónico en promover la salud de la mama. Objetivo: Explorar la relación que existe entre conocimientos y comportamientos sobre factores de riesgo y detección oportuna del cáncer de mama en un grupo de mujeres. Material y métodos: Estudio piloto observacional, descriptivo y transversal, incluyó 52 mujeres mayores de 25 años de una institución educativa. Las variables fueron: factores de riesgo para CaMa, antecedentes personales, conductas protectoras o de riesgo, conocimientos y screening, Resultados: con una $K$ Richardson 0.73. La media de edad 38 \pm 8 , las mujeres de 47-57 años tienen menor nivel de escolaridad ( $Z=-2.87, p=0.004$ ), y menos conocimientos (Anova $p=0.02$ ). Las mujeres que no reportaron datos de enfermedad crónica tienen un promedio mayor de conductas de riesgo $(3 \pm 1)$ que aquellas que si lo presentan $(2 \pm 1, t=2.944$, $g \mid=50, p<0.005)$. Discusión: Los resultados concuerdan con lo reportado en la literatura, los conocimientos son deficientes y no se relacionan con conductas saludables ni garantizan que las mujeres realicen detección oportuna. Conclusiones: En el espacio universitario se deben implementar estrategias que incrementen los conocimientos y la detección oportuna de CaMa.

Este artículo se deriva del Proyecto Titulado: Nivel de conocimientos sobre factores de riesgo para cáncer de mama en estudiantes de enfermería de pregrado: Diseño e implementación de una intervención educativa. Aprobado por el Comité de Investigación de la ENEO, Registro: 046 (20101) Responsable de la Investigación: Mtra. Sofía Elena Pérez Zumano

-Profesora Asociada B de TC. Unidad de Investigación ENEO. UNAM.

..Pasantes de la Licenciatura en Enfermería y Obstetricia.

...Profesora Asociada B de TC. Sistema de Universidad abierta ENEO. UNAM.

Correspondencia: sepzumano2@yahoo.com.mx

RECIBIDO: 29 DE OCTUBRE DE 2010. ENVIADO: 5 DE NOVIEMBRE DE2010. ACEPTADO: 1 DICIEMBRE DE 2010. 
Introduction: In Mexico, every two hours a woman dies of breast cancer (BrCa); thus Nursing should assume a principal roll in the promotion of women's health. objective: explore the relationship between knowledge and behavior related to risk factors and BrCa early detection. Materials and methods: basic, descriptive, and observational study pilot, which included 52 women older than 25 working in an education institution. The variables were BrCa risk factors, personal background, protecting or risk conducts, knowledge and screening. Results: K Richardson was .73. Average age was $38+-8$. Women between 47-57 reported a lower level of education ( $Z=-2.87, p=.004)$, and less knowledge (ANOVA $p=.02)$. Women who did not report chronic illness data reported greater average number of risk conducts $(3+-1)$ than those who did $(2+-1, t=2.944, g)=50, p<.005)$. Discussion: our results are consistent with those of the literature: when knowledge is insufficient women do not behave healthy and do not perform early detection practices. Conclusion: it is necessary to implement strategies to enhance knowledge and frequency of BrCa early detection practices.

\section{INTRODUCCIÓN}

El cáncer de mama (CaMa) es la causa más común de muerte por neoplasia en las mujeres de todo el mundo con más de 410,000 defunciones por año, casi $14 \%$ de todas las muertes debidas al cáncer en las mujeres. ${ }^{1}$

En México en el 2006 murieron 4,451 mujeres por CaMa, es decir un fallecimiento cada dos horas. El $60 \%$ de las mujeres muere entre los 30 y 59 años de edad, ${ }^{2}$ en plena etapa productiva y muchas en reproductiva. Por lo menos dos terceras partes de las muertes en mujeres menores de 75 años se pudieran evitar, si se hiciera una detección temprana y un tratamiento oportuno se salvarían 11,000 vidas. $^{3}$

La mayoría de los casos es autodetectable y solo entre el $5 \mathrm{y}$ el $10 \%$ se diagnostica en los estadios 0 y I. ${ }^{4}$ Las mujeres que son diagnosticadas en estadio I tienen un $98 \%$ de sobrevida y mayores probabilidades de mantenerse libres de la enfermedad a diferencia de aquéllas que son diagnosticadas en estadios más avanzados. ${ }^{5}$
Lo anterior evidencia la magnitud del problema y la necesidad de trabajar en la promoción de la salud de la mama y en la detección oportuna de factores de riesgo y/o conductas relacionados con el incremento o disminución del CaMa.

Los factores de riesgo de mayor peso para desarrollar CaMa son el género y la edad, además de éstos la NOM 041-SSA2-2002 señala: vida menstrual de más de 40 años; menarquía temprana (antes de los 12 años), menopausia tardía (después de los 52 años), nuliparidad, primer embarazo a término después de los 30 años de edad, terapia hormonal de reemplazo (mayor de 5 años), historia familiar de CaMa, enfermedad benigna de los senos y obesidad. ${ }^{6-9}$

También existe evidencia que el síndrome metabólico tiene relación con la prevalencia e incidencia de CaMa. ${ }^{10-11}$

Por otra parte, también hay factores modificables del estilo de vida, que sin ser específicos para desarrollar CaMa se han relaciona- do con el incremento de éste, tales como: dieta alta en hidratos de carbono y en carga glicémica, consumo excesivo de carnes rojas, grasas saturadas y pobre ingesta de frutas y vegetales, además de consumo de alcohol, sedentarismo, sobrepeso u obesidad. ${ }^{10-13}$

Por el contrario, se ha demostrado que cuando la ingesta de lípidos es adecuada y el consumo de verduras es abundante, la dieta se convierte en un factor protector.

Existe fuerte evidencia de que verduras, frutas, cereales integrales, fibra dietética, ciertos micronutrientes, algunos ácidos grasos protegen contra algunos cánceres. ${ }^{16}$

También la actividad física es un factor protector ya que cambia los biomarcadores para riesgo de cáncer, mejora el bienestar físico y psicológico y se asocia a otras conductas saludables como dieta y el control de peso. ${ }^{14-21}$

Es sobre estos factores modificables del estilo de vida en los que se debe trabajar de manera oportuna para reducir la incidencia del 
CaMa. En este contexto, es innegable la responsabilidad de la institución universitaria formadora de profesionales de Enfermería de transformar la cultura y el cuidado de la salud de las personas, lo cual se debe iniciar con las mujeres que cotidianamente participan de la dinámica institucional ya sean alumnas o trabajadoras, promoviendo los conocimientos de salud de la mama, la identificación y modificación de comportamientos relacionados con la enfermedad.

Existe evidencia que las mujeres que poseen conocimientos sobre la salud de la mama conocen las características normales de éstas y tienen mayores probabilidades de detectar una lesión y solicitar atención inmediata si perciben algo anormal. ${ }^{23-24}$

En un estudio en mujeres universitarias se evaluó el conocimiento sobre el CaMa, los resultados arrojaron que la mayoría de ellas tenían conocimientos inadecuados, sin embargo, más de dos tercios de las estudiantes conocían las recomendaciones del autoexamen y de examen clínico de mama. ${ }^{25}$

En otro estudio se evaluó el nivel de conocimientos y la frecuencia en la práctica de exámenes de detección oportuna de cáncer cérvico uterino y de mama, respecto al CaMa se descubrió que más de la mitad de las mujeres $56.4 \%$ tienen conocimientos sobre esta enfermedad, sin embargo, cuando se evalúo el conocimiento y la práctica de autoexploración de mama se encontró que ambos son bajos y la principal causa es el desconocimiento de la técnica. ${ }^{26}$

Con relación a los comportamientos existen diversos reportes de investigación que demuestran que los conocimientos acerca de la salud no son suficientes para modificar el estilo de vida, por ejemplo en una muestra de 207 trabajadores del primer nivel de atención, se encontró una prevalencia de $46 \%$ de sobrepeso y obesidad principalmente en médicos $y$ laboratoristas. ${ }^{27}$

De hecho existen hallazgos que muestran que los profesionistas de la salud tienen mayores porcentajes de prácticas nocivas o al menos no anticipatorias para la salud en comparación con otros profesionistas universitarios. $^{28}$

Algo similar puede estar ocurriendo en las instituciones formadoras de profesionales de la salud donde se ha identificado un predominio de comportamientos poco saludables entre sus estudiantes. ${ }^{29}$

Por el contrario, en otro estudio se exploró la relación de los estilos de vida con la calidad de vida de los estudiantes de los Programas Educativos de psicología, nutrición, odontología, cultura física y deportes, que reveló que existen beneficios recíprocos entre salud y educación. ${ }^{30}$

Esta información es relevante, ya que con las mujeres que laboran en la institución educativa y las jóvenes estudiantes; futuras profesionales de la salud, se pueden trabajar estrategias de modificación del estilo de vida que disminuyan el riesgo de enfermedades crónicas, que contribuyan a su bienestar y que les proporcionen bases para un cuidado de la salud congruente.

En el país existe poca evidencia en la disciplina de enfermería acerca del nivel de conocimientos que poseen las estudiantes y personal que labora en la institución educativa por lo que el objetivo

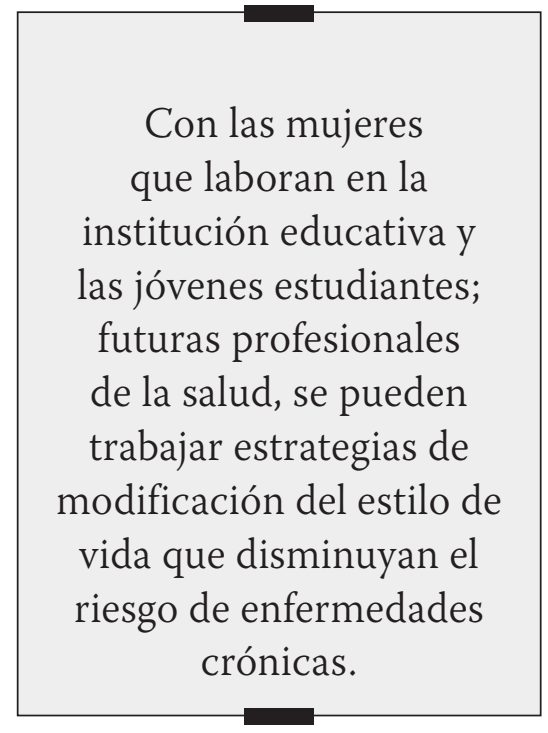

del estudio fue explorar la relación que existe entre conocimientos y comportamientos sobre factores de riesgo y detección oportuna del cáncer de mama en un grupo de mujeres.

\section{Metodología}

Se realizó un estudio observacional, prolectivo, descriptivo y transversal como prueba piloto en una institución educativa. El instrumento fue elaborado exprofeso para este estudio con base en la revisión de la literatura y fue sometido a validez de contenido por un grupo de expertos.

Además de los datos sociodemográficos se exploran las variables: antecedentes personales, factores de riesgo para CaMa con base en la Norma Oficial Mexicana NOM-041-SSA2-2002, conductas protectoras o de riesgo (tabaquismo, alcoholismo, actividad física, alimentación e información $\mathrm{CaMa}$ ), prácticas de screening (autoexploración, exploración clínica y mastografía) y conocimientos.

El índice de masa corporal se calculó con autorreporte de peso y talla y autoperpeción de la imagen corporal mediante la selección 
de la silueta que mejor representara la imagen de la mujer, en una ilustración de 9 siluetas, en rangos que van desde muy delgadas hasta extremadamente obesas. ${ }^{31}$ Así mismo, se implementó la escala de actividad física (IPAQ-International

Physical Activity Questionnarie) validado y utilizado para la población latina. ${ }^{32}$

La confiabilidad del instrumento es aceptable con un $\mathrm{K}$ de $\mathrm{Ri}$ chardson de $r=.073$

La muestra estuvo constituida por 52 mujeres mayores de 25 años que aceptaron participar. Para llevar a cabo la investigación se tomó en cuenta los principios éticos de beneficencia y no maleficencia, veracidad, privacidad y confidencialidad, señalados en el código ético de Enfermería y los relativos al Reglamento de la Ley General de Salud.

La recolección de datos se obtuvo con el consentimiento informado de las mujeres. Durante este proceso se brindó una explicación de los objetivos del estudio y se les aclaró sus dudas, haciéndoles énfasis que el empleo de la información sería completamente confidencial.

Los datos se analizaron en el paquete estadístico SPSS versión 15.

\section{Resultados}

La población de estudio estuvo constituida por 52 mujeres; trabajadoras (30.8\%), estudiantes (61.5\%) y docentes (7.7\%), el promedio de edad fue de $38 \pm 8$. De acuerdo a la edad de la mujer se conformaron tres grupos, el porcentaje más alto correspondió al grupo de 25 a 35 años con un total de $42 \%$.

El 48\% de las mujeres son casadas o están en unión libre, 35\% solteras y un $17 \%$ divorciadas o separadas. El nivel máximo de escolaridad fue el medio superior (bachillerato-carrera técnica) con un $58 \%$, las mujeres con menor nivel de escolaridad son las de 47 a 57 años $(\mathrm{Z}=-2.87, \mathrm{p}=0.004)$ y además son las que menos saben acerca del CaMa $(7 \pm 3)$, a diferencia de las mujeres de 25 a $35(10 \pm 4)$ y las de 36 a 46 (12 \pm 4$)$, llama la atención que a mayor edad menor nivel de conocimientos a pesar de que el riesgo es mayor. Figuras 1, 2.

Al analizar el nivel de conocimientos con las variables ocupación y nivel de estudios no se encontraron diferencias.
Respecto a la frecuencia de factores de riesgo para $\mathrm{CaMa}$ en el grupo de estudio se encontró que el 23\% de mujeres tiene antecedente familiar, el 39\% más de 40 años, el (17\%) padece alguna enfermedad benigna de los senos. Casi la mitad de nuestra población se encuentra con problemas de peso; $37 \%$ sobrepeso y $11 \%$ obesidad.

Los factores de riesgo para CaMa se sumaron para crear una nueva variable como riesgo total y fue analizada con diversas variables.

Referente a las enfermedades crónicas se encontró que más de

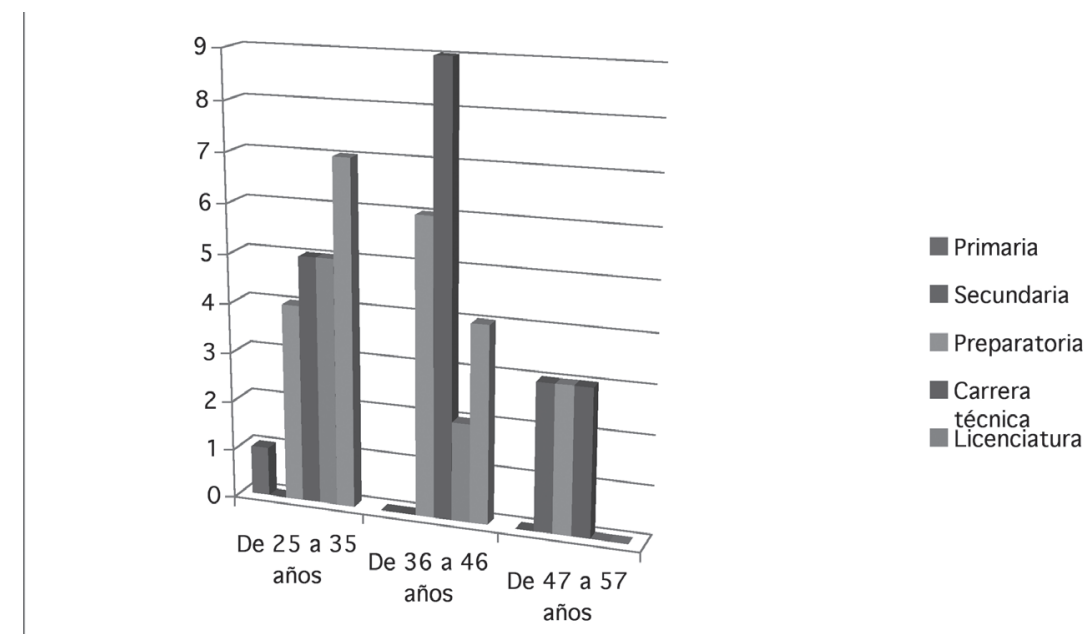

Proporción de mujeres de acuerdo a nivel educativo por grupo de edad.

Figura 1. Kruskal-Wallis con prueba posthoc U de Mann Whitney $\mathrm{p}<0.004$.

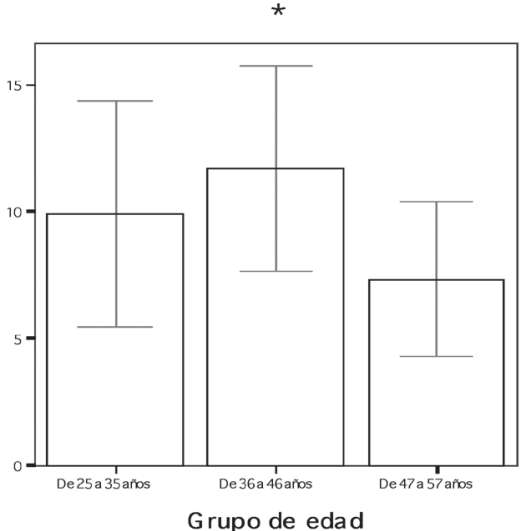

Figura 2.

Puntaje de conocimientos por grupo de edad (puntaje máximo 22). ANOVA con prueba de posthoc Tukey $\mathrm{p}=0.029$

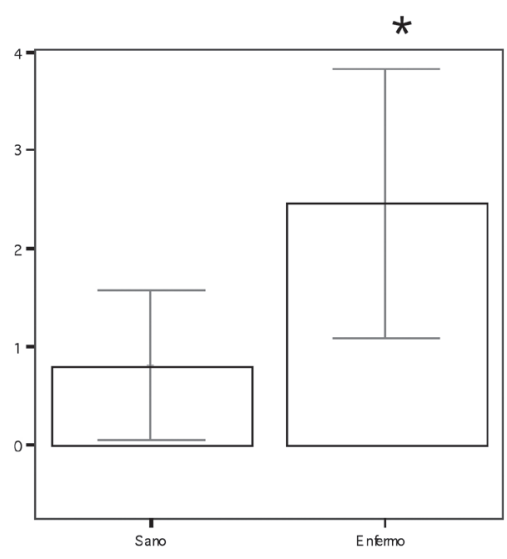

Figura 3. Clasifcación Dx

Suma de factores de riesgo para desarrollar Cáncer de Mama en mujeres sanas y enfermas. Prueba t de Student " $\mathrm{p}<0.0001$ 
la mitad de las mujeres reportó por lo menos un dato de síndrome metabólico: diabetes mellitus (8\%), hipercolesterolemia (25\%) e hipertriglicéridemia (19\%), con este autoreporte se agruparon en sanas y enfermas, encontrándose que las enfermas tienen más factores de riesgo para desarrollar CaMa $(2 \pm 1)$ que las sanas $(1 \pm 1)(\mathrm{t}=-5.113$, $\mathrm{gl}=30.44, \mathrm{p}<0.0001)$. Figura 3.

Con relación a los comportamientos el 10\% de las mujeres fuman, 42\% mencionó no realizar ninguna actividad física, sin embargo, con base a los criterios de la escala internacional de actividad física se clasificó como sedentaria al 98\%, el 50\% ingiere bebidas alcohólicas y $83 \%$ tienen una alimentación deficiente en calidad y cantidad. Estos datos aluden que la mayoría de las entrevistadas no tienen comportamientos saludables a pesar de que casi 7 de cada 10 son personal de salud.

Al comparar el promedio de conductas del estilo de vida se encontró que las mujeres sanas tienen un promedio más alto de comportamientos de riesgo $(3 \pm 1)$ que las enfermas $(2 \pm 1) .(t=2.944, g l=50, p$

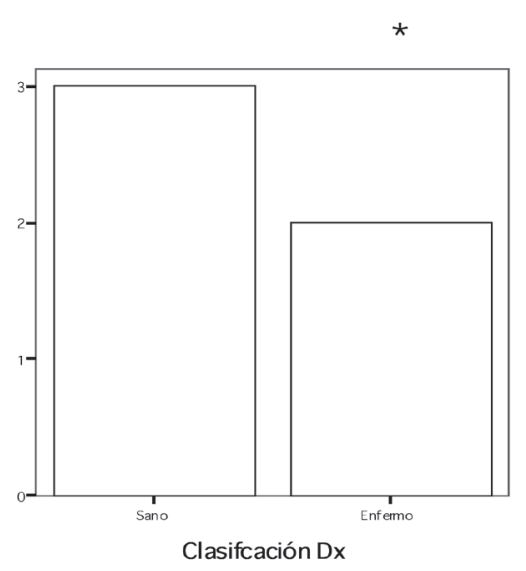

Figura 4.

Suma de estilos de vida por diagnóstico. U de Mann-Whitney "p $<0.005$
$<0.005)$, este hallazgo sugiere que las mujeres enfermas tienen más comportamientos saludables. Figura 4.

Respecto a las conductas de screening con CaMa no se encontraron diferencias en la práctica de autoexamen y mastografía, pero si en el examen clínico donde el 88\% de mujeres menores de 39 años reportan no haber acudido a la unidad de salud para realizárselo $\left(\mathrm{x}^{2}\right.$ $\mathrm{p}=0.01$ ).

Solo el 13\% mencionó contar con información de CaMa.

Por último, no se encontró relación entre el nivel de conocimientos y los comportamientos protectores para CaMa. $\left(r_{p}=-0.076, p>0.05\right)$

\section{Discusión}

Aunque no existen medidas de protección específica para el CaMa, la evidencia señala diversos aspectos del estilo de vida que influyen sobre la incidencia y prevalencia de la enfermedad, se esperaría que las mujeres trabajadoras o estudiantes de una escuela de enfermería tuvieran un buen nivel de conocimientos y comportamientos protectores para el CaMa.

Sin embargo, en este estudio se encontró que los conocimientos que poseen son deficientes, lo que concuerda con otros reportes de investigación donde se han evaluado diversos aspectos del CaMa encontrando que la mayoría de las mujeres tienen conocimientos insuficientes, además que existe incongruencia entre lo que dicen saber con la práctica correcta del autoexamen de senos..$^{25-26}$

Las mujeres de más de 47 años con menor nivel de escolaridad y de conocimientos, son las más vulnerables del grupo estudiado, por lo
La institución educativa debe crear una cultura de salud en sus estudiantes, por lo que hay que revisar los programas del plan de estudios para identificar que contenidos y actividades de aprendizaje hay sobre la salud de la mama.

que es urgente implementar intervenciones de enfermería que contribuyan a solucionar esta situación en las que se considere como variables intervinientes la edad, la residencia y la escolaridad que determinan de manera particular la práctica de autoexamen.

La edad y el género son los principales factores de riesgo no modificables en las mujeres estudiadas, sin embargo, el sobrepeso u obesidad se presenta en casi la mitad de ellas y éste es un factor relacionado directamente con la incidencia de la enfermedad $1^{1,18}$ lo anterior se explica al analizar los comportamientos relacionados con la dieta y actividad física que reveló malos hábitos alimenticios y sedentarismo en la mayoría de las mujeres estudiadas, hallazgos semejantes se han encontrado en estudios con profesionales de la salud y estudiantes universitarios, una vez más se revela que los comportamientos son paradójicos con los conocimientos que se poseen. ${ }^{27-29}$

Además, es relevante destacar que a pesar de ser una población joven varias mujeres ya presentan datos de síndrome metabólico directamente relacionado con la dieta y la actividad física que incremen- 
tan significativamente el riesgo de presentar CaMa, sin embargo, son éstas mujeres las que más conductas saludables presentan en comparación con las mujeres sanas. En ambos casos es necesario establecer estrategias de intervención que promuevan la salud integral de las mujeres, para la reducción de factores de riesgo de enfermedades crónicas en particular el cáncer de mama que hoy día es un problema de salud pública.

\section{Conclusiones}

La institución educativa debe crear una cultura de salud en sus estudiantes, por lo que hay que revisar los programas del plan de estudios para identificar que contenidos $y$ actividades de aprendizaje hay sobre la salud de la mama que preparen a los alumnos para la prevención y detección oportuna del CaMa, ya que ellos como agentes de cambio pueden promoverla en sí mismos, en su familia y personas a quienes proporcionan cuidados, además de hacerla extensiva a las trabajadoras contribuyendo así a la disminución de la incidencia y detección oportuna del CaMa.

\section{REFERENCIAS}

1 Anderson B, Shyyan R, Eniu AE, Smith R, Yip C, Bese NS, et al.El cáncer de mama en los países con Recursos limitados: Sinopsis de las Normativas del 2005 de la Iniciativa Mundial de Salud de la Mama. The Breast Journal Suplemento 2007; 13 (1):S16-29.

2 Lozano R, Knaul FM, Gómez Dantés, Arreola-Ornelas y Méndez O. Tendencias en la mortalidad por cáncer de mama en México, 19792006. Observatorio de la Salud. Documento de trabajo. Competi- tividad y Salud, Fundación Mexicana para la Salud,2008.

3 Franco-Marina F, Lozano R, Villa B, Solís P. La mortalidad en México, 2000-2004. Muertes evitables: magnitud, distribución y tendencias. Dirección General de Información en Salud, Secretaría de Salud México, 2006.

4 Secretaría de Salud. Programa de Acción Cáncer de Mama. México 2002; p.62.

5 Lester J. Breast Cancer in 2007: Incidence, Risk Assessment, and Risk Reduction Strategies. Clinical Journal Oncology Nursing. 2007; 11(5):619-23.

6 McPherson K, Steel CM, Dixon JM. Breast cancer-epidemiology, risk factors, and genetics. British Medical Journal. 2000. 321; 624-8.

7 Lacovara JE, Ray J. Deciphering the Diagnostics of Breast Cancer. MEDSURG Nursing. 2007; 16(6):391-410.

8 Cárdenas J, Sandoval F. Coord. Consenso Nacional sobre diagnóstico y tratamiento del cáncer mamario. Tercera Revisión. Colima (México): Masson Doyma, 2009.

9 NOM 041-SSA2-2002, Para la prevención, diagnóstico, tratamiento, control y vigilancia epidemiológica del cáncer de mama. Disponible en http://www.salud.gob.mx/unidades/cdi/nom/041ssa202.html. consultada el 10 de marzo 2011.

10 Furberg AS, Veierød MB,Wilsgaard T, Bernstein L,Thune I. Serum High-Density Lipoprotein Cholesterol, Metabolic Profile, and Breast Cancer Risk. Journal of the National Cancer Institute 2004; 96(15): 1152-60.

11 Bray G. The underlying basis for obesity: Relationship to cancer. The Journal of Nutrition. 2002; 132 (11S):3451-5.
12 Romieu I, Lazcano E, Sanchez LM, Willett W, Hernandez M. Carbohydrates and the Risk of Breast Cancer among Mexican Women. Cancer Epidemiology, Biomarkers \& Prevention. 2004; 13(8):1283-9.

13 Stephenson G, Rose D. Breast Cancer and Obesity: An Update. Nutrition and Cancer. 2003; 45(1):2-17.

14 Renehan A, Tyson M, Egger M, Heller R, Zwahlen M. Body-mass index and incidence of cancer: a systematic review and metaanalysis of prospective observational studies. The Lancet. 2008; 371 (9612): 569-78.

15 Romieu I, Lajous M. The role of obesity, physical activity and dietary factors on the risk for breast cancer: Mexican experience. Salud Publica Mex 2009; 51 (suppl 2): S172-S180.

16 Torres Sánchez L, Galván Portillo M, Lewis S, Gómez Dantes H, López Carrillo L. Dieta y cáncer de mama en Latinoamerica. Salud Publica Mex 2009; 51(supl 2):s181-s190.

17 Campbell K, McTierman. Exercise and Biomarkers for Cancer Prevention Studies The Journal of Nutrition .2007; S137(1):161-170.

18 Adderly KB, Williams E. The Relations Between Obesity and Breast Cancer. ABNF Journal. 2003; 61-5.

19 Rock CH, Demark-Wahnefried W. Can lifestyle modification increase survival in women diagnosed with breast cancer. The Journal of Nutrition. 2002; 132 (11S): S3504-5.

20 Pinto BM, Floyd A. Methodologic issues in exercise intervention research in oncology. SeminOncolNurs. 2007; 23 (4): 297-304.

21 Friedenreich C, Orenstein M. Physical activity and cancer prevention: Etiologic evidence and bio- 
logical mechanisms. The Journal of Nutrition. 2002; 132, (11S) : S3456-64.

22 Ortiz-SP, Torres G, Mainero F, Ángeles A, López AE, Lazcano E, Romieu I. Actividad física y riesgo de cáncer de mama en mujeres mexicanas. Consultado 10 enero 09. Disponible en http://www.infodoctor.org:8080/uid=18372993

23 Ortega D, López L, López M. Estrategias para la enseñanza del autoexamen de seno a mujeres en edad reproductiva. Salud Publica de Mex 2000; 42 (1): 17-25.

24 Martínez-Montañez OG, UribeZuñiga P, Hernández-Ávila M. Políticas públicas para la detección del cáncer de mama en México. Salud Publica Mex. 2009; 51(2):S350S360.

25 Hadi MA, Hassali M, Shafie AA, Awaisu A. Evaluation of breast cancer awareness among female university students in Malaysia. Pharmacy Practice;2008 8(1):2934.
26 Cumpián-Loredo B. Conocimiento sobre detección oportuna de $\mathrm{CaCu}$ y mamario. Hospital General de Zona 11. Piedras Negras, Coahuila. Instituto Mexicano del Seguro Social, Rev Enferm. IMSS 2000; 8 (3):129-32.

27 Palacios-Rodríguez R, MunguíaMiranda C, Ávila-Leyva A. Sobrepeso y obesidad en personal de salud de una unidad de medicina familiar. Rev Med Inst Seguro Soc 2006; 44 (5): 449-53.

28 Hernández I, Arenas LM, Martínez PC, Menjívar A. Autocuidado en profesionistas de la salud y profesionistas universitarios. Acta Universitaria, 2003; 13 (3): 26-32. Disponible en: http:// redalyc.uaemex.mx/redalyc/ pdf/416/41613302.pdf Consultado el 18 junio 2010

29 Moreno E, Vázquez L, Gutiérrez G, Martínez L, Quevedo M, González M, Salas G. Estudio piloto sobre prevalencia de obesidad en universitarios mexicanos y hábi- tos de salud relacionados. International Journal of Pshychology and Psychological Therapy. 2004; 4(3): 623-638.

30 Meda R, De Santos F, Lara B, Verdugo J, Palomera A, Valadez MD. Evaluación de la percepción de calidad de vida y el estilo de vida en estudiantes desde el contexto de las Universidades Promotoras de la Salud. Revista de Educación y Desarrollo 2008; 8: 5-16.

31 Osuna-Ramírez I, Hernández-Prado B, Campuzano JC, Salmerón J. Índice de masa corporal y percepción de la imagen corporal en una población adulta mexicana: la precisión del autorreporte. Salud Publica Mex 2006; 48(2): 49-103.

32 Peña Quimbaya E, Colina Gallo E, Vásquez Gómez AC. Actividad física en empleados de la universidad de Caldas, Colombia. Hacia la promoción de la salud. 2009; 14(2): $52-65$. 